\title{
Valuation of Electricity Swing Options by Multistage Stochastic Programming
}

\author{
Gido Haarbrücker ${ }^{a}$, Daniel Kuhn ${ }^{\mathrm{b}, \star}$ \\ ${ }^{a}$ Institute for Operations Research and Computational Finance (ior/cf-HSG), University of St. Gallen, Switzerland \\ ${ }^{\mathrm{b}}$ Department of Computing, Imperial College London, United Kingdom
}

\begin{abstract}
Electricity swing options are Bermudan-style path-dependent derivatives on electrical energy. We consider an electricity market driven by several exogenous risk factors and formulate the pricing problem for a class of swing option contracts with energy and power limits as well as ramping constraints. Efficient numerical solution of the arising multistage stochastic program requires aggregation of decision stages, discretization of the probability space, and reparameterization of the decision space. We report on numerical results and discuss analytically tractable limiting cases.
\end{abstract}

Key words: Stochastic programming; Model approximation; Discretization; Energy; Finance; Swing option

\section{Introduction}

The ongoing deregulation of electricity markets worldwide has a major impact on the power industry. New price risks require new risk management tools and new methods for the valuation of generation and transmission assets as well as physical and financial electricity contracts. As far as risk management is concerned, many derivative instruments have been designed to hedge against spot price risk or different types of liability risk exposure. For instance, one observes the emergence of markets for standard derivatives such as futures and European call and put options. Examples of such derivative markets comprise the European Energy Exchange (EEX), Amsterdam Power Exchange (APX), Nord Pool with Eltermin and Eloption, and New York Mercantile Exchange (NYMEX). In addition, there is an immense variety of derivative contracts with Bermudan-style and path-dependent payoff structure; these contracts are usually traded over-the-counter. Probably the most important examples are swing options, which are sometimes also referred to as take-or-pay contracts, virtual

\footnotetext{
* Corresponding author.
}

Please cite this article as: Haarbrücker, G., \& Kuhn, D. Valuation of electricity swing options by multistage stochastic programming, Automatica (2009), doi: 10.1016/j.automatica.2008.11.022

Email addresses: gido.haarbruecker@unisg.ch (Gido Haarbrücker), dkuhn@doc.ic.ac.uk (Daniel Kuhn).

Article published in Automatica (2009), 45:4, pp. 889-899 power plants, base-load factor contracts, or flexible nomination contracts. A swing option is an agreement to purchase and or sell electric energy during a fixed period of time and at a predetermined price. The option holder may choose her consumption process freely within certain restrictions stipulated in the contract. Usually, there is some flexibility in the timing and amount of consumption. Swing options are tailored for risk-averse agents willing to mitigate (spot) price risk. As electrical energy is storable only in a limited manner, the timing flexibility is appreciated by agents who are unable to control their electricity consumption. While simple futures contracts provide no timing and volumetric flexibility at all, a strip of standard call and or put options could offer more flexibility than needed, thus involving too high option premia; see e.g. Jaillet, Ronn, and Tompaidis (2004). Therefore, swing options represent ideal hedging instruments, e.g., for risk-averse public utilities facing a stochastic load demand. More information on the role of swing options in risk management is provided by Doege, Schiltknecht, and Lüthi (2006) who demonstrate how swing options or storage power plants can be used to hedge against adverse movements of a power portfolio. Finally, swing options are also used as speculative instruments.

Virtually any existing valuation scheme for swing option contracts can be traced back to a stochastic dynamic programming (SDP) approach. For instance, Thompson (1995) develops an SDP scheme within a lattice 
whereas Jaillet, Ronn, and Tompaidis (2004) work with a forest of recombining trees. Lari-Lavasanni, Simchi, and Ware (2001) present a similar discrete forest methodology together with a proof of convergence and various sensitivity analyses. On the other hand, Davison and Anderson (2003) consider specific swing options on weekly average on-peak electricity prices. They estimate optimal exercise boundaries and determine the option value via subsequent Monte Carlo simulation. Other promising valuation schemes are based on the Least Squares Monte Carlo approach (LSM) due to Longstaff and Schwartz (2001), see e.g. Ibáñez (2004). The LSM algorithm uses least squares regression to estimate the conditional expectation functions involved in the SDP iterations. An alternative Monte Carlo method for the valuation of swing options is discussed in Meinshausen and Hambly (2004), and a linear programming based lower bound on the swing option value is suggested by Keppo (2004). Carmona and Touzi (2008) as well as Carmona and Dayanik (2008) study the optimal multiple stopping problem (inherent in the swing option pricing problem) by using methods of stochastic calculus. Closely related is the optimal switching problem that arises in the valuation of gas storage facilities, which share many of the distinctive properties of swing options, see e.g. Carmona and Ludkovski (2005).

This article addresses challenging swing option pricing problems that are difficult to solve with SDP techniques. The reason for this difficulty is threefold. Firstly, we embed the valuation problem in a model economy driven by several risk factors. Multi-dimensionality of risk is a key feature of any realistic market model as it eliminates perfect correlation of the forward price fluctuations corresponding to different maturities. Secondly, we consider swing options involving ramping constraints, i.e., we impose restrictions on the gradient of the consumption rate; see Section 3. Notice that standard SDP schemes, as e.g. in Jaillet, Ronn, and Tompaidis (2004), Lari-Lavasanni, Simchi, and Ware (2001), and Thompson (1995), are designed to deal with swing contracts involving limitations on the consumption rate and its integral, but not on its gradient. However, ramping constraints are important when a swing option (virtual power plant) is used to mimic a real power plant with limited start-up speed as described in Thompson, Davison, and Rasmussen (2004). Thirdly, we study contracts with many exercise times such as hourly exercisable swing options over one year. Numerical experiments suggest that SDP and its variants are well adapted for pricing daily exercisable swing options without ramping constraints and given that risk is one-dimensional. For the pricing problems addressed in this article, SDP does not provide sufficiently accurate solutions in reasonable time.

Due to suitable aggregation of decision stages and discretization of the risk space, our stochastic programming approach entails short computation times in the range of only few seconds. Moreover, calculations are based on the current forward price curve. Thus, our valuation scheme meets typical operational requirements of trading and risk management departments in privatized electricity companies: decisions on whether submitting or accepting an offer have usually to be taken within a time frame of some minutes only, and exercise decisions should always take account of the latest market data.

This article is structured as follows. Section 2 develops a Pilipovic-type model for the forward price dynamics which fully characterizes the electricity market under consideration. In Section 3, we specify the swing option contracts to be considered in this work and propose an exact valuation scheme. Next, Section 4 provides some a priori information on the option's value and optimal exercise strategy in different zones of parameter space, while Section 5 approximates the exact pricing problem by a numerically tractable auxiliary problem. Section 6 reports on numerical results, and Section 7 concludes.

\section{Forward Price Dynamics}

When tackling the valuation of electricity swing options, one faces several major difficulties. First, electricity cannot be stored efficiently, and production has to cover demand instantaneously. Therefore, the traditional, storage based no-arbitrage methods of valuing commodity derivatives fail. Moreover, markets for electricity derivatives suffer from insufficient liquidity; there are large bid-offer spreads, and agents face a significant counterparty risk. In addition, electricity spot prices are meanreverting and exhibit strong seasonalities, jumps, spikes, and regime-switching behavior. These complicating features result from the non-storability and the grid bound nature of power. Finally, swing options are non-standard instruments with Bermudan-style and path-dependent payoff structure. Hence, sophisticated valuation schemes are required.

In this article, we will assume the electricity spot and forward markets to be arbitrage-free, frictionless, and efficient since their liquidity is likely to rise in the future. However, we do not make the unrealistic assumption that electrical energy is storable or traded. A market is arbitrage-free if and only if there exists a risk-neutral probability measure $Q$ under which the discounted prices of all investment goods are martingales. These investment goods must be traded, that is, their prices must be determined by matching demand and supply in a liquid market, and they must be storable. The latter requirement is trivially satisfied for securities, but for commodities it only holds in a limited manner. Since electricity forwards are securities, their discounted prices are in fact martingales under the risk-neutral measure $Q$. In contrast, the discounted spot price of electricity is generally not a martingale because electricity constitutes a non-storable commodity. In the sequel, we assume that 
the electricity forwards represent a set of basis securities, which can be used to hedge more complicated electricity derivatives, e.g. swing options. We further stipulate that these basis securities define a complete market, which means that the underlying martingale measure $Q$ is unique. In this setting, any contingent claim on electricity can be replicated by dynamically trading in the forwards and a risk-free instrument, for more details see e.g. Deng, Johnson, and Sogomonian (1998), Section 3. The law of one price then dictates that the contingent claim and the corresponding hedging portfolio have the same price at any time. For simplicity of exposition, throughout this article we will assume that the risk-free interest rate is zero. Note that this assumption could easily be relaxed at the cost of additional notation.

Let us now specify the probabilistic model which underlies the swing option pricing problem to be formulated in Section 3. Since electricity traders' decisions are usually based on current (and historic) forward price information, we should provide a model for the forward price dynamics. Our approach is inspired by the popular Pilipovic model, Pilipovic (1998), Section 5.6, which is widely used in the energy sector and has the advantage of analytical tractability. Note that the classical Pilipovic model describes the spot price dynamics of energy commodities. Here, however, a related model for the forward prices is proposed. Concretely speaking, we will work with a probability space $(\Omega, \mathcal{F}, Q)$ on which a two-dimensional Brownian motion $\boldsymbol{B}_{t}=\left(B_{1, t}, B_{2, t}\right)$ is defined. Recall that the components of $\boldsymbol{B}_{t}$ are independent one-dimensional Brownian motions. For technical details we refer to Protter (1990). Next, we let $\{F(0, T)\}_{T \geq 0}$ be the deterministic forward price curve observed at time 0 . For any $0 \leq t \leq T$, we will assume that the forward price $F(t, T)$ at time $t$ is represented by the random variable

$F(t, T)=F(0, T) \frac{E\left(\exp \xi_{1, T} \mid \mathcal{F}_{t}\right)}{E\left(\exp \xi_{1, T}\right)}$,

where $\boldsymbol{\xi}_{t}=\left(\xi_{1, t}, \xi_{2, t}\right)$ is a vector of risk factors subject to the stochastic differential equation (SDE)

$d \boldsymbol{\xi}_{t}=A \boldsymbol{\xi}_{t} d t+\Sigma d \boldsymbol{B}_{t}$,

and $\mathcal{F}_{t}=\sigma\left(\boldsymbol{\xi}_{s} \mid 0 \leq s \leq t\right)$ is the $\sigma$-algebra generated by the history of risk factors up to time $t$. Furthermore, the matrices appearing in (2) are given by

$A=\left(\begin{array}{cc}-\alpha_{1} & \alpha_{1} \\ 0 & -\alpha_{2}\end{array}\right) \quad$ and $\quad \Sigma=\left(\begin{array}{cc}\sigma_{1} & 0 \\ 0 & \sigma_{2}\end{array}\right)$

where $\alpha_{1}>\alpha_{2} \geq 0$ and $\sigma_{1}, \sigma_{2}>0$. These parameters have to be estimated empirically. Although the calibration of the forward price model under consideration is beyond the scope of the present article, we should remark that one usually finds $\alpha_{1} \gg \alpha_{2}$ and $\sigma_{1} \gg \sigma_{2}$ in real energy markets. Therefore, the second risk factor $\xi_{2, t}$ can conveniently be interpreted as the equilibrium level around which $\xi_{1, t}$ fluctuates. Systems of SDEs of the type (2) can be solved systematically: with initial data $\boldsymbol{\xi}_{s}, 0 \leq s \leq t$, the solution reads

$$
\boldsymbol{\xi}_{t}=\underbrace{\exp (A(t-s))}_{=H_{t, s}} \boldsymbol{\xi}_{s}+\underbrace{\int_{s}^{t} \exp (A(t-r)) \Sigma d \boldsymbol{B}_{r}}_{=\boldsymbol{\varepsilon}_{t, s}}
$$

where 'exp' stands for the matrix exponential, i.e.,

$\exp (A t)=\left(\begin{array}{cc}e^{-\alpha_{1} t} & \frac{\alpha_{1}}{\alpha_{1}-\alpha_{2}}\left(e^{-\alpha_{2} t}-e^{-\alpha_{1} t}\right) \\ 0 & e^{-\alpha_{2} t}\end{array}\right)$.

The random variable $\varepsilon_{t, s}$ is independent of the outcome history $\left\{\boldsymbol{\xi}_{r}\right\}_{r \leq s}$. From elementary stochastic calculus we know that $\varepsilon_{t, s}$ is normally distributed, i.e.,

$\varepsilon_{t, s} \sim \mathcal{N}\left(0, \int_{s}^{t} \exp (A(t-r)) \Sigma \Sigma^{\top} \exp (A(t-r))^{\top} d r\right)$.

Substituting the first component of $\boldsymbol{\xi}_{T}=H_{T, t} \boldsymbol{\xi}_{t}+\boldsymbol{\varepsilon}_{T, t}$ into the formula for the forward prices (1), calculating the conditional expectation value, and applying Itô's lemma we obtain the SDE

$$
\begin{aligned}
& \frac{d F(t, T)}{F(t, T)}=e^{-\alpha_{1}(T-t)} \sigma_{1} d B_{1, t} \\
& \quad+\frac{\alpha_{1}}{\alpha_{1}-\alpha_{2}}\left(e^{-\alpha_{2}(T-t)}-e^{-\alpha_{1}(T-t)}\right) \sigma_{2} d B_{2, t} .
\end{aligned}
$$

Observe that the first Brownian motion accounts for the fluctuations of the short-term forward prices $\left(\alpha_{1}^{-1} \gg\right.$ $T-t \geq 0$ ), whereas the second Brownian motion determines the mid-term fluctuations $\left(\alpha_{2}^{-1} \gg T-t \gg \alpha_{1}^{-1}\right)$. Thus, there is no perfect correlation between the forward price fluctuations across the different maturities, which is an important feature of multifactor models. The spot price of electricity can be written as

$S(t)=F(t, t)=F(0, t) \frac{\exp \xi_{1, t}}{E\left(\exp \xi_{1, t}\right)}$.

As manifested by (1) and (5), the forward price process $\{F(t, T)\}_{0<t<T}$ is indeed a martingale with respect to the filtered probability space $\left(\Omega, \mathcal{F},\left\{\mathcal{F}_{t}\right\}_{t \geq 0}, Q\right)$ for each fixed maturity date $T$, whereas the spot price process $\{S(t)\}_{t \geq 0}$ is not. Substituting $\boldsymbol{\xi}_{T}=H_{T, 0} \boldsymbol{\xi}_{0}+\boldsymbol{\varepsilon}_{T, 0}$ into (1), we can verify that the distribution of the forward prices is independent of the choice of the initial data $\boldsymbol{\xi}_{0}$. Without loss of generality, the start value $\boldsymbol{\xi}_{0}$ may thus be set to zero, which will always be assumed from now on. Moreover, by substituting $\boldsymbol{\xi}_{T}=H_{T, t} \boldsymbol{\xi}_{t}+\boldsymbol{\varepsilon}_{T, t}$ into (1), we find that there is a one-to-one correspondence between forward price curves and risk factors in 
our model economy. In other words, for fixed $t$, each vector of risk factors $\boldsymbol{\xi}_{t}$ uniquely determines a forward price curve $\{F(t, T)\}_{T \geq t}$ and vice versa. There is also a one-to-one correspondence between spot prices and the first components of the risk factors: for fixed $t$, each $\xi_{1, t}$ uniquely determines a spot price $S(t)$ and vice versa. However, as the spot price represents one single point on the forward price curve, it is not possible to reconstruct $\boldsymbol{\xi}_{t}$ from only observing $S(t)$. These results will be important when modelling the information structure underlying the swing option pricing problem.

In practice, one might wish to work with more realistic multivariate forward price models involving jumps and spikes, regime switching, and stochastic volatility. In these cases, our methodology for pricing swing options to be developed in Section 3 still applies.

\section{$3 \quad$ Electricity Swing Options}

This section introduces the instrument 'electricity swing option' and sketches the range of swing option types under consideration. In brief, an electricity swing option is an agreement to purchase and or sell electrical energy at a predetermined strike price $K$ during a fixed contract period $[\underline{t}, \bar{t}]$, and it provides some flexibility in the quantity to be delivered; see Kaminski and Gibner (1995), Barbieri and Garman (1996), or Pilipovic and Wengler (1998). Typically, a swing option appears along with limitations on power purchased (or sold) at time $t$ and energy accumulated within the interval $[\underline{t}, t]$, where $t$ ranges from $\underline{t}$ to $\bar{t}$. In general practice, limits on energy quantities relate to the whole contract period. Thus, cumulative energy at time $\bar{t}$ is required to lie between the given target values $\underline{e}$ and $\bar{e}$. In the case where underor over-shooting these limits is allowed, penalties $c^{-}$or $c^{+}$are imposed on each unit of shortfall or exceedance, respectively. Note that energy constraints corresponding to shorter subperiods can also be handled. However, because of their rare occurrence, such constraints will be disregarded in the sequel. Limitations on power hold at any time $t$ in the contract period. The power level may only be changed at discrete time points $t_{i}=i \Delta$, $i \in \mathcal{I}=\{\underline{i}, \ldots, \bar{i}\} \subset \mathbb{Z}$, stipulated in the swing contract. Thus, power is chosen freely at each time $t_{i}$, based on available forward price information, and remains fixed at some level $p_{i}$ in the interval from $t_{i}$ to $t_{i+1}, i \in \mathcal{I}$. The contractual parameter $\Delta$ characterizes the length of a rebalancing interval. Typically, $\Delta$ lies between 15 minutes and several hours. We require the start and end dates of the contract period to be compatible with the subdivision into rebalancing intervals, i.e., $\underline{t}=\underline{i} \Delta$ and $\bar{t}=(\bar{i}+1) \Delta$. Furthermore, swing options are assigned a quality. One usually distinguishes base, peak, and offpeak quality, allowing the option rights to be exercised only in base, peak, or off-peak hours within the contract period, respectively. This feature is conveniently
Table 1

Contractual parameters

\begin{tabular}{clr}
\hline Param. & Value & Unit \\
\hline$\underline{t}$ & start of the delivery period & $(\mathrm{h})$ \\
$\bar{t}$ & end of the delivery period & $(\mathrm{h})$ \\
$\Delta$ & length of a rebalancing interval & $(\mathrm{h})$ \\
$K$ & strike price & $(€ / \mathrm{MWh})$ \\
$\left\{\underline{p}_{i}\right\}_{i \in \mathcal{I}}$ & lower power limits & $(\mathrm{MW})$ \\
$\left\{\bar{p}_{i}\right\}_{i \in \mathcal{I}}$ & upper power limits & $(\mathrm{MW})$ \\
$p_{\text {start }}$ & start power & $(\mathrm{MW})$ \\
$\underline{e}$ & lower energy limit at expiry $\bar{t}$ & $(\mathrm{MWh})$ \\
$\bar{e}$ & upper energy limit at expiry $\bar{t}$ & $(\mathrm{MWh})$ \\
$\left\{\varrho_{i}\right\}_{i \in \mathcal{I}}$ & ratchets & $(\mathrm{MW})$ \\
$c^{+}$ & penalty costs for exceeding $\bar{e}$ & $(€ / \mathrm{MWh})$ \\
$c^{-}$ & penalty costs for undershooting $\underline{e}$ & $(€ / \mathrm{MWh})$ \\
\hline
\end{tabular}

captured by introducing time-dependent power limits:

$\underline{p}_{i} \leq p_{i} \leq \bar{p}_{i} \quad \forall i \in \mathcal{I}$.

Sometimes, additional ramping constraints are specified. These constraints limit the slope of the load pattern corresponding to a given exercise strategy. The maximum power difference $\varrho_{i} \geq 0$ between rebalancing intervals $i-1$ and $i$ is referred to as a ratchet, see e.g. Barbieri and Garman (1996). Usually, ratchets are constant over time. However, imagine that the rebalancing interval has length one hour, and one wishes to price a daily exercisable swing option: then, one may set up a model with $\varrho_{i}=0$ unless $i$ is a multiple of 24 . In the absence of ramping constraints we simply set $\varrho_{i}=\varrho_{\max , i}=$ $\max \left\{\bar{p}_{i-1}-\underline{p}_{i}, \bar{p}_{i}-\underline{p}_{i-1}\right\}$ for all $i \in \mathcal{I}$. Thus, formally speaking, we may always require

$\left|p_{i}-p_{i-1}\right| \leq \varrho_{i} \quad \forall i \in \mathcal{I}$

Here, the dummy variable $p_{i-1}$ is set to the start power $p_{\text {start }}$, which represents another contractual parameter. Typically, swing contracts involving nontrivial ratchets (which are strictly smaller than $\varrho_{\max , i}$ ) are assigned base quality; hence, the option is exercisable at any time in the contract period. However, ramping constraints can also be meaningful when dealing with 'non-base' quality. The complete parameter set necessary to characterize a swing contract is shown in Table 1 . The decision variables at the choice of the option holder are $\left\{p_{i}, e_{i}\right\}_{i \in \mathcal{I}}$. As mentioned above, $p_{i}$ stands for power in the $i$ th rebalancing interval, whereas $e_{i}$ denotes the amount of energy exercised by the end of interval $i$.

Without loss of generality, let us now determine the price of a given swing option at time $t=0$ (today). It is always assumed that $\bar{t}>0$, but we will consider problems with $\underline{t} \geq 0$ as well as $\underline{t}<0$. In the latter case, we determine 
the residual option value corresponding to the remaining delivery period from 0 to $\bar{t}$ and contingent on the amount of energy exercised in the period from $\underline{t}$ to 0 . Valuation is based on the following assumptions: agents are rational and greedy, they have access to efficient electricity spot and forward markets, the forward price dynamics is governed by the SDE (4), there are no bid-ask spreads or transaction costs, no discounting takes place over time, and electrical energy is not storable. We will work directly under the martingale measure $Q$. Recall that the electricity market under consideration is complete, implying that $Q$ is in fact unique. Consequently, the unique no-arbitrage price of the swing contract equals the sum of the expected cash flows transferred from the issuer to the holder. However, these cash flows depend on the option holders' exercise strategy. The challenge of pricing a swing contract is thus to evaluate the option holder's exercise strategy under the premise that he or she is a rational agent trying to maximize the contract value. Note that in an incomplete market there would be a whole interval of no-arbitrage prices.

For the sake of transparent notation, we define $\mathcal{I}^{+}$as the index set of the future rebalancing intervals, $\mathcal{I}^{+}=\mathcal{I} \cap \mathbb{N}_{0}$. Moreover, in the remainder we will always choose time units such that $\Delta=1$. At time 0 , the option holder observes the forward price curve $\{F(0, T)\}_{T \geq 0}$ and, bearing in mind the contractual constraints, decides on the exercise level $p_{0}$, which is held constant up to time 1 . Thus, the contract issuer is obliged to sell the holder an amount of energy $p_{0}$ at the strike price $K$. As the agents cannot accumulate energy, they square positions instantaneously in the spot market. At time 1, a new forward price curve $\{F(1, T)\}_{T \geq 1}$ is observed, in response to which the contract holder chooses the exercise level $p_{1}$, etc. This procedure recurs until the end of the delivery period. Generally speaking, at time $i \in \mathcal{I}^{+}$, the net cash flow $(S(i)-K) p_{i}$ is transferred from the issuer to the contract holder. The exercise decisions $p_{i}$ and $e_{i}$ may depend on forward price information observed at times $j=0, \ldots, i$; however, they are required to be independent of all information revealed at later rebalancing intervals $j=i+1, \ldots, \bar{i}$. This property is referred to as non-anticipativity in literature, see e.g. Birge and Louveaux (1997). In Section 2 we have argued that there is a one-to-one correspondence between forward price curves and risk factors. Thus, we may equivalently state that, for $i \in \mathcal{I}^{+}$, the decisions $p_{i}$ and $e_{i}$ depend exclusively on information about the risk factors $\left\{\boldsymbol{\xi}_{j}\right\}_{j=0}^{i}$. As the risk factors are random variables on $(\Omega, \mathcal{F}, P)$, so are the decisions. By non-anticipativity, they are $\mathcal{G}_{i}$-measurable, where $\mathcal{G}_{i}=\sigma\left(\boldsymbol{\xi}_{j} \mid j=0, \ldots, i\right)$ is the sub- $\sigma$-algebra (or the information set) generated by the risk factors observed in the past. ${ }^{1}$ At this point of progress, it is sat-

\footnotetext{
1 The random variable $p_{i}:(\Omega, \mathcal{F}) \rightarrow(\mathbb{R}, \mathcal{B}(\mathbb{R}))$ is $\mathcal{G}_{i^{-}}$ measurable if and only if there is a measurable function $\varphi_{i}:\left(\mathbb{R}^{2(i+1)}, \mathcal{B}\left(\mathbb{R}^{2(i+1)}\right)\right) \rightarrow(\mathbb{R}, \mathcal{B}(\mathbb{R}))$ such that $p_{i}=$
}

isfactory to establish the optimization problem for valuing swing options in a formal way. The development of a computationally tractable mathematical model is postponed to Section 5. The (residual) value of a swing option at time $t=0$ is given by the optimal value of the following multistage stochastic program (cf. Birge and Louveaux (1997), Section 3.5):

$$
\begin{array}{ll}
\max & E \sum_{i \in \mathcal{I}^{+}}(S(i)-K) p_{i} \\
\text { s.t. } & \underline{e} \leq e_{\bar{i}} \leq \bar{e} \\
& e_{i}-e_{i-1}=p_{i} \\
& \underline{p}_{i} \leq p_{i} \leq \bar{p}_{i} \\
& \left|p_{i}-p_{i-1}\right| \leq \varrho_{i} \\
& p_{i}, e_{i} \mathcal{G}_{i} \text {-measurable }
\end{array}
$$

Thus, we maximize the option holders' expected (future) profit earned by means of the (future) exercise strategy $\left\{p_{i}, e_{i}\right\}_{i \in \mathcal{I}^{+}}$. Observe that the energy balance equation (or continuity equation) determines the relation between power and cumulative energy. If $\underline{t}<0$ (i.e., 'today' lies within the delivery period), then $e_{-1}$ represents the amount of energy exercised from $\underline{t}$ to 0 , and $p_{-1}$ stands for initial power in interval -1 . Otherwise, if $\underline{t} \geq 0$ (i.e., the delivery period lies in the future), then the dummy variable $e_{\underline{i}-1}$ is set to zero while $p_{\underline{i}-1}$ is set to $p_{\text {start }}$. All admissible exercise strategies comply with the contractual constraints on power and energy as well as with the ramping constraints. As already mentioned above, we require the decisions $p_{i}$ and $e_{i}$ to be non-anticipative with respect to the underlying information process $\left\{\mathcal{G}_{i}\right\}_{i \in \mathcal{I}^{+}}$.

Note that, although the forward prices (or equivalently: the risk factors) do not show up explicitly in the stochastic program (6), they impact the pricing problem through the important measurability constraint. If the option holder could observe only spot prices instead of the entire forward price curves, we should require the time- $i$ decisions to be measurable with respect to $\sigma\left(\xi_{1, j} \mid j=0, \ldots, i\right)$, which is a real sub- $\sigma$-algebra of $\mathcal{G}_{i}$. This extra restriction would reduce the swing option value. However, since electricity traders do in fact observe the entire forward price curves, we can work with the 'richer' information process $\left\{\mathcal{G}_{i}\right\}_{i \in \mathcal{I}^{+}}$.

Notice that the energy limits are strict in (6), i.e., failure to meet the energy targets is penalized with an infinite loss. Passing over to finite penalties is straightforward but omitted for better readability. We will briefly return to the case of finite penalties in the next section.

$\varphi_{i}\left(\boldsymbol{\xi}_{0}, \ldots, \boldsymbol{\xi}_{i}\right)$; see e.g. Ash (1972), Theorem 6.4.2 (c). Nonanticipativity is obvious from the last expression. 


\section{A Priori Analysis}

For ease of exposition, consider a swing option contract with strict energy limits and without ramping constraints, i.e., $\varrho_{i}=\varrho_{\max , i}$ for all $i \in \mathcal{I}$. Without performing any calculations, exact statements are available about the optimal exercise strategy and the option value in specific regions of parameter space. To see this, consider again the stochastic program (6). It has relatively complete recourse, see e.g. Rockafellar and Wets (1976), if we explicitly take account of the induced constraints

$\underline{e}-\sum_{j=i+1}^{\bar{i}} \bar{p}_{j} \leq e_{i} \leq \bar{e}-\sum_{j=i+1}^{\bar{i}} \underline{p}_{j} \quad \forall i \in \mathcal{I}^{+}$.

These constraints will play a decisive role in the below discussion. Fig. 1 sketches the time-energy diagram of the swing option under consideration, and the inset at the bottom shows the corresponding quality pattern. Precisely speaking, the grey bars indicate on-quality intervals when the option may principally be exercised (here we show the situation $\bar{p}_{i}>\underline{p}_{i}>0$ ), and the spaces denote off-quality intervals $\left(\bar{p}_{i} \stackrel{-\underline{p}}{=} \underline{p}_{i}=0\right)$. In all onquality intervals, the option holder should purchase energy at a rate no lower than $\underline{p}_{i}$ and no larger than $\bar{p}_{i}$, whereas in all off-quality intervals energy is held constant. Observe that points outside the shaded region are not accessible when starting with zero energy at the beginning of the contract period.

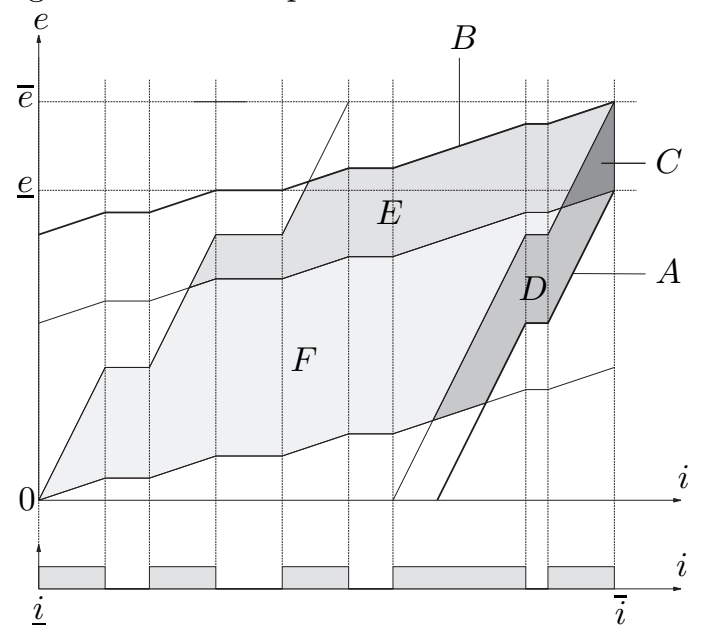

Fig. 1. Time-energy diagram

The time-energy plane is divided into different zones, where analytical a priori statements about the optimal exercise strategy $\left\{p_{i}^{*}\right\}_{i \in \mathcal{I}^{+}}$(Table 2 ) and the option value (Table 3) can be made. Notice that the 'upper' and 'lower' induced constraints (7) are binding in zones $A$ and $B$, respectively. Therefore, the option has to be exercised at maximum power in zone $A$. Otherwise, the target value $\underline{e}$ would be undershot by the end of the contract period. Conversely, the swing option has to be ex-
Table 2

Optimal exercise strategy in different regions of parameter space ('n/a' indicates that there is no a priori statement)

\begin{tabular}{cl}
\hline Region & Optimal Strategy at time $i$ \\
\hline$A$ & $p_{i}^{*}=\bar{p}_{i}$ \\
$B$ & $p_{i}^{*}=\underline{p}_{i}$ \\
$C$ & $S(i) \geq K \Rightarrow p_{i}^{*}=\bar{p}_{i} ; S(i)<K \Rightarrow p_{i}^{*}=\underline{p}_{i}$ \\
$D$ & $S(i) \geq K \Rightarrow p_{i}^{*}=\bar{p}_{i} ; S(i)<K \Rightarrow \mathrm{n} / \mathrm{a}$ \\
$E$ & $S(i) \leq K \Rightarrow p_{i}^{*}=\underline{p}_{i} ; S(i)>K \Rightarrow \mathrm{n} / \mathrm{a}$ \\
$F$ & $\mathrm{n} / \mathrm{a}$ \\
\hline
\end{tabular}

Table 3

Option value in different regions of parameter space

\begin{tabular}{cl}
\hline Region & Option Value at time $i$ \\
\hline$A$ & $=\sum_{j=i}^{\bar{i}} \bar{p}_{j}(F(i, j)-K)$ \\
$\mathrm{B}$ & $=\sum_{j=i}^{\bar{i}} \underline{p}_{j}(F(i, j)-K)$ \\
$C$ & $=\sum_{j=i}^{\bar{i}} \underline{p}_{j}(F(i, j)-K)+\left(\bar{p}_{j}-\underline{p}_{j}\right) C(i, j)$ \\
$D$ & $\geq \sum_{j=i}^{\bar{i}} \bar{p}_{j}(F(i, j)-K)$, \\
& $\leq \sum_{j=i}^{\bar{i}} \underline{p}_{j}(F(i, j)-K)+\left(\bar{p}_{j}-\underline{p}_{j}\right) C(i, j)$ \\
$E$ & $\geq \sum_{j=i}^{\bar{i}} \underline{p}_{j}(F(i, j)-K)$, \\
& $\leq \sum_{j=i}^{\bar{i}} \underline{p}_{j}(F(i, j)-K)+\left(\bar{p}_{j}-\underline{p}_{j}\right) C(i, j)$ \\
$F$ & $\leq \sum_{j=i}^{\bar{i}} \underline{p}_{j}(F(i, j)-K)+\left(\bar{p}_{j}-\underline{p}_{j}\right) C(i, j)$ \\
\hline
\end{tabular}

ercised at minimum power in zone $B$ in order to prevent exceedance of the energy target $\bar{e}$ at expiry. In any case, the option reduces to a portfolio of cash and forward contracts with different maturities.

In zone $C$ both the upper and lower energy limits become obsolete. The optimal exercise level at time $i$ only depends on the sign of $S(i)-K$, and the swing option reduces to a portfolio of cash, forwards, and European call options with different maturities. We denote by $C(i, j)$ the time- $i$ price of a European call option which pays off $(S(j)-K)^{+}$at time $j \geq i$.

In zones $D$ and $E$ the upper and lower energy limits, respectively, become redundant. Some implications of this partial redundancy are summarized in Tables 2 and 3 . Note that the upper bounds on the option value are obtained by relaxation of the energy constraints. In contrast, the lower bound in $D$ is due to feasibility of $\left\{p_{i}=\bar{p}_{i}\right\}_{i \in \mathcal{I}^{+}}$, while the lower bound in $E$ is due to feasibility of $\left\{p_{i}=\underline{p}_{i}\right\}_{i \in \mathcal{I}^{+}}$.

Next, consider zone $F$ where no precise a priori statement about the optimal decisions is available. Nevertheless, studying the dynamic version of the stochastic program (6) provides qualitative insights. The current shadow price of energy is defined as the rate of change of the option value with respect to small changes of the parameter $e_{-1}$, i.e., the energy exercised in the past. In the limit of (infinitesimally) short rebalancing intervals one 
can prove that the option should be exercised at maximum power if the shadow price of energy is larger or equal to the difference of the strike and the spot price. Otherwise, it is recommended that the option be exercised at minimum power. Note that determination of the shadow price of energy requires solution of the pricing problem (6). The upper bound on the option value is again by relaxation of the energy constraints.

If exceeding or undershooting energy limits is allowed, it is in principle possible to leave the shaded region, and the above qualitative statements should be reconsidered. In case of high penalty costs, the above results can serve as a first approximation. However, we find that $S(i)>K+c^{+}$ implies $p_{i}^{*}=\bar{p}_{i}$, and $S(i)<K-c^{-}$implies $p_{i}^{*}=\underline{p}_{i}$ independent of the volume exercised in the past. In the presence of ramping constraints, the above qualitative analysis has to be generalized. Allowing for nontrivial ratchets leads to a power dependence of the induced constraints (7). To obtain similar results as above, one would have to extend the time-energy diagram of Fig. 1 by adding the dimension of power. However, as it reveals hardly any new insights, there is no need to go through this tedious analysis here.

\section{Approximations}

For later reference we will refer to the stochastic program (6) as the exact pricing problem. In a complete market, the optimal value of (6) represents the maximum amount of money which can be earned from the underlying swing option contract without risk. Realization of this certain profit requires implementation of the optimal exercise strategy and continuous delta hedging. ${ }^{2}$

Unfortunately, the stochastic program (6) cannot be solved numerically. In fact, each decision variable represents a random object with a continuum of realizations. Thus, (6) must be viewed as an optimization problem over an infinite-dimensional function space, which is computationally untractable. The standard approach to tackle this problem is to discretize the underlying probability space. But then, one still fails to deal with the large number of decision stages, which may amount to 8760 for hourly rebalanced swing options with a typical contract period of one year. In order to establish an approximate pricing problem, which can be solved numerically, we successively perform three basic approximations:

1) reduction of the information process;

2) discretization of the probability space;

3) reduction of the number of decision variables.

\footnotetext{
2 A market is complete if all risks are traded in the sense that investors can obtain any desired exposure to the shocks to the economy. Notice that continuous delta hedging requires the risks to be traded (and observed) continuously.
}

In the remainder of this section, we discuss these basic approximations and assess the errors thereby incurred.

\subsection{Reduction of the Information Process}

The exact pricing problem (6) typically has $\left|\mathcal{I}^{+}\right|>500$ decision stages, each of which is assigned two decision variables. By reducing the underlying information process, we effectively establish a new optimization problem with only few decision stages, each of which is assigned several hundred decision variables. Concretely speaking, the future contract period from today's perspective, i.e., the interval from $0 \vee \underline{t}$ to $\bar{t}$, is divided into a number of periods indexed by $s \in \mathcal{S}^{+}=\{0, \ldots, \bar{s}\}$. These periods must be of (possibly different) integer lengths. For each $s \in \mathcal{S}^{+}$, let $i_{s}$ be the first rebalancing interval belonging to period $s$, and set $i_{\bar{s}+1}=\bar{i}+1$. Usually, we have $\left|\mathcal{S}^{+}\right| \ll\left|\mathcal{I}^{+}\right|$implying that each period covers many rebalancing intervals. The exercise strategy or 'load pattern' within period $s$ is given by the following vector of power decisions $\boldsymbol{p}_{s}=\left(p_{i_{s}}, \ldots, p_{i_{s+1}-1}\right) \in \mathbb{R}^{d_{s}}$, where $d_{s}=i_{s+1}-i_{s}$. With a slight abuse of notation we denote by $e_{s}$ the amount of energy exercised until the end of stage $s$. Of course, by virtue of the energy balance equation, $e_{s}$ is fully determined by the preceding power decisions. For the sake of transparent notation, we define $C_{s}$ as the set of load patterns in period $s$ which comply with the power and ramping constraints, i.e.,

$$
\begin{aligned}
& C_{s}=\left\{\boldsymbol{p}_{s} \in \mathbb{R}^{d_{s}}: \underline{p}_{i} \leq p_{i} \leq \bar{p}_{i} \text { for } i_{s} \leq i<i_{s+1}\right. \text { and } \\
& \left.\left|p_{i}-p_{i-1}\right| \leq \varrho_{i} \text { for } i_{s}<i<i_{s+1}\right\} \text {. }
\end{aligned}
$$

By construction, $C_{s}$ is convex polyhedral and compact. Next, introduce a $d_{s}$-dimensional random vector $\boldsymbol{S}_{s}=\left(S\left(i_{s}\right), S\left(i_{s}+1\right), \ldots, S\left(i_{s+1}-1\right)\right)$, and let $\mathbf{1}_{m}=(1, \ldots, 1) \in \mathbb{R}^{m}$ for all $m \in \mathbb{N}$. With these conventions, the amount of energy exercised within period $s$ is given by $\left\langle\mathbf{1}_{d_{s}}, \boldsymbol{p}_{s}\right\rangle$, while the profit earned in period $s$ amounts to $\left\langle\boldsymbol{S}_{s}-K \mathbf{1}_{d_{s}}, \boldsymbol{p}_{s}\right\rangle$. As a first important approximation, we assume now that forward price updates are no longer observed at all intervals $i \in \mathcal{I}^{+}$but only at intervals $i \in\left\{i_{s} \mid s \in \mathcal{S}^{+}\right\} \subset \mathcal{I}^{+}$. Thus, from now on the option holder's decisions $\boldsymbol{p}_{s}$ and $e_{s}$ will depend on the information $\mathcal{H}_{s}$ available at period $s$ :

$\mathcal{H}_{s}=\sigma\left(\boldsymbol{\xi}_{i_{r}} \mid r=0, \ldots, s\right), \quad s \in \mathcal{S}^{+}$

By definition, $\mathcal{H}_{s}$ is a real sub- $\sigma$-algebra of $\mathcal{G}_{i}$ for all $i \geq i_{s}$, and the approximate pricing problem associated with the reduced information process $\left\{\mathcal{H}_{s}\right\}_{s \in \mathcal{S}^{+}}$is given 
by the multistage stochastic program

$$
\left.\left.\begin{array}{ll}
\max & E \sum_{s \in \mathcal{S}^{+}}\left\langle\boldsymbol{F}_{s}-K \mathbf{1}_{d_{s}}, \boldsymbol{p}_{s}\right\rangle \\
\text { s.t. } & \underline{e} \leq e_{\bar{s}} \leq \bar{e} \\
& \boldsymbol{p}_{s} \in C_{s} \\
& \left\langle\mathbf{1}_{d_{s}}, \boldsymbol{p}_{s}\right\rangle=e_{s}-e_{s-1} \\
& \boldsymbol{p}_{s}, e_{s} \mathcal{H}_{s} \text {-measurable }
\end{array}\right\} \forall s \in \mathcal{S}^{+}\right\} Q \text {-a.s. }
$$

Here, we used the law of iterated conditional expectations to replace $\boldsymbol{S}_{s}$ by the random vector $\boldsymbol{F}_{s}=E\left(\boldsymbol{S}_{s} \mid \mathcal{H}_{s}\right)$. Energy target constraints are as in (6), while power and ramping constraints are formulated abstractly by using the compact sets $\left\{C_{s}\right\}_{s \in \mathcal{S}^{+}}$. However, notice that the approximate pricing problem (8) disregards the ramping constraints at each rebalancing interval $i_{s}$ for $s \in \mathcal{S}^{+}$. This deficiency could principally be remedied at the cost of additional notation, but the gain in precision would be modest since $\left|\mathcal{S}^{+}\right| \ll\left|\mathcal{I}^{+}\right|$. As usual, neighboring decision stages are coupled by the energy balance equation, and the dummy variable $e_{-1}$ stands for the amount of energy exercised in the past. In other words, energy purchased (sold) until the end of period $s$ must equal energy purchased (sold) until the end of period $s-1$ and energy purchased (sold) in period $s$. In contrast to the exact pricing problem (6), the decisions $\boldsymbol{p}_{s}$ and $e_{s}$ are required to be non-anticipative with respect to the reduced information process $\left\{\mathcal{H}_{s}\right\}_{s \in \mathcal{S}^{+}}$.

\subsection{Discretization of the Probability Space}

The approximate pricing problem (8) remains computationally intractable as it exhibits infinitely many scenarios. The standard approach to overcome this difficulty is to discretize the underlying probability space. In doing so, one effectively approximates the stochastic program (8) by an optimization problem over a finite-dimensional Euclidean space, which is numerically tractable.

The selection of an appropriate discrete probability measure $Q^{d}$ is referred to as scenario generation and represents a primary challenge in the field of stochastic programming. A survey and critical assessment of modern scenario generation techniques is provided in Kaut and Wallace (2007). Once a suitable discrete measure $Q^{d}$ is identified, we approximate (8) by the stochastic program

$$
\left.\left.\begin{array}{ll}
\max & E^{d} \sum_{s \in \mathcal{S}^{+}}\left\langle\boldsymbol{F}_{s}-K \mathbf{1}_{d_{s}}, \boldsymbol{p}_{s}\right\rangle \\
\text { s.t. } & \underline{e} \leq e_{\bar{s}} \leq \bar{e} \\
& \boldsymbol{p}_{s} \in C_{s} \\
& \left\langle\mathbf{1}_{d_{s}}, \boldsymbol{p}_{s}\right\rangle=e_{s}-e_{s-1} \\
& \boldsymbol{p}_{s}, e_{s} \mathcal{H}_{s^{-}} \text {-measurable }
\end{array}\right\} \forall s \in \mathcal{S}^{+}\right\} Q^{d} \text {-a.s. }
$$

Here, $E^{d}(\cdot)$ denotes expectation with respect to $Q^{d}$. There is a vast number of scenario generation methods for which the difference of the optimal values of problems (8) and (9) can be estimated in a quantitative sense. Socalled bounding methods (cf. Birge and Louveaux (1997), Chap. 9) allow for a strict estimate of the approximation error whereas sampling-based methods (cf. Birge and Louveaux (1997), Chap. 10) provide a probabilistic estimate. Sometimes, the difference of the optimal solution sets can be estimated, too; see e.g. Pflug (1996), Section 1.3, or Kuhn (2004), Sections 4.6 and 5.5. Usually, the approximation can be improved by adding new atoms to the discrete measure $Q^{d}$.

The numerical calculations in Section 6 are based on a moment matching method proposed in Siede (2000), Section 7.3. This particular scenario generation technique approximates each conditional distribution of the risk factors in the next stage by a transformed trinomial distribution with $k$ trials and uniform event probabilities $\left(\frac{1}{3}, \frac{1}{3}, \frac{1}{3}\right) . k \in \mathbb{N}$ plays the role of a tuning parameter which determines the branching factor of the arising scenario tree, $(k+2)(k+1) / 2$. The branching factor, which is constant throughout the tree, is defined as the number of branches emanating from each non-terminal tree node. Recall that trinomial distributions have only two independent components since the sum of all three components must be equal to $k$. Thus, there exists an affine mapping which transforms the trinomial distribution to a discrete bivariate distribution whose first and second order moments match those of the risk factors. This transformed trinomial distribution is used to construct the scenario tree. By the central limit theorem, it converges weakly to the true conditional distribution of the risk factors as $k$ tends to infinity. This convergence result relies on the fact that the risk factors are normally distributed; see (3). As it is specially tailored to normal distributions, the described moment matching technique leads to very accurate option prices in our computational tests in Section 6.

\subsection{Reduction of the Number of Decision Variables}

After reducing the information process and discretizing the probability space, the resulting stochastic program (9) principally allows for numerical solution. However, in order to speed up computation time, we should reduce the number of decision variables. We begin with a suitable reparameterization of the load patterns in the compact set $C_{s}$. To this end, let $\left\{\boldsymbol{p}_{s}^{n}\right\}_{n=1}^{N_{s}}$ be the vertices of $C_{s}$. Then, any vector $\boldsymbol{p}_{s} \in C_{s}$ is representable as a convex combination of the form

$\boldsymbol{p}_{s}=\sum_{n=1}^{N_{s}} \boldsymbol{p}_{s}^{n} \lambda_{s}^{n}, \quad \sum_{n=1}^{N_{s}} \lambda_{s}^{n}=1, \quad \lambda_{s}^{n} \geq 0 \quad \forall n$.

Notice that any $\boldsymbol{p}_{s} \in C_{s}$ can be viewed as an exercise profile for period $s$, which satisfies the power and ramping 
constraints. In this sense, the vertices of $C_{s}$ constitute extremal exercise profiles. Every exercise profile can be represented as a weighted sum of the extremal profiles with convex weights $\left\{\lambda_{s}^{n}\right\}_{n=1}^{N_{s}}$. Conversely, every convex combination of the extremal profiles yields an exercise profile in $C_{s}$, and thus it satisfies the power and ramping constraints. However, the representation (10) is generally not unique, i.e., there may be different sets of convex weights for the same exercise profile.

So far, our reparameterization has no numerical advantages: in fact, we have even increased the number of decision variables since the dimension of $C_{s}$ is usually much smaller than the number of its vertices $\left(d_{s} \ll N_{s}\right)$. The key observation is that we are not interested in representing all exercise profiles in $C_{s}$ but only those which are potentially optimal in the approximate pricing problem (9). As we will argue below, it is sufficient to concentrate on few vertices $\left\{\boldsymbol{p}_{s}^{n}\right\}_{n=1}^{n_{s}}\left(n_{s} \ll d_{s}\right)$. By this means we can substantially reduce the number of decision variables in period $s$.

In order to formulate the pricing problem in the proposed 'exercise profile framework', we need the following definitions:

$$
\begin{aligned}
\boldsymbol{c}_{s} & =\left(c_{s}^{1}, \ldots, c_{s}^{n_{s}}\right), \quad c_{s}^{n}=\left\langle\boldsymbol{F}_{s}-K \mathbf{1}_{d_{s}}, \boldsymbol{p}_{s}^{n}\right\rangle, \\
\boldsymbol{w}_{s} & =\left(w_{s}^{1}, \ldots, w_{s}^{n_{s}}\right), \quad w_{s}^{n}=\left\langle\mathbf{1}_{d_{s}}, \boldsymbol{p}_{s}^{n}\right\rangle \\
\boldsymbol{\lambda}_{s} & =\left(\lambda_{s}^{1}, \ldots, \lambda_{s}^{n_{s}}\right),
\end{aligned}
$$

with $\boldsymbol{c}_{s}$ an exogenous random vector, $\boldsymbol{w}_{s}$ a constant vector, and $\boldsymbol{\lambda}_{s}$ representing a decision variable for every $s \in \mathcal{S}^{+}$. Using the above conventions, problem (9) can be approximated by the reparameterized stochastic program

$$
\begin{aligned}
& \max E^{d} \sum_{s \in \mathcal{S}^{+}}\left\langle\boldsymbol{c}_{s}, \boldsymbol{\lambda}_{s}\right\rangle \\
& \text { s.t. } \underline{e} \leq e_{\bar{s}} \leq \bar{e}
\end{aligned}
$$

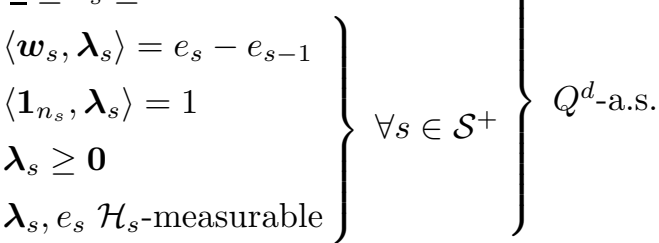

Note that the power limits and ramping constraints are still satisfied in (11) since the load pattern corresponding to a feasible weight vector $\boldsymbol{\lambda}_{s}$ is a convex combination of some vertices of the convex set $C_{s}$, thus being an element of $C_{s}$, as well. If we take account of all vertices associated with the sets $\left\{C_{s}\right\}_{s \in \mathcal{S}^{+}}$, then (11) is equivalent to (9). Neglecting certain vertices associated with conjecturally suboptimal exercise strategies restricts the feasible set. Thus, the maximum of problem (11) is never larger than (but hopefully close to) the maximum of problem (9).
In order to keep the gap between the maxima of (9) and (11) small, we need a heuristics to select a small number $n_{s}$ of suitable extremal exercise profiles (that is, vertices of $C_{s}$ ). A good heuristics is obtained by considering the dynamic version of problem (9), which reveals that the optimal load pattern $\boldsymbol{p}_{s}$ maximizes the sum of an immediate certain profit $\left\langle\boldsymbol{F}_{s}-K \mathbf{1}_{d_{s}}, \boldsymbol{p}_{s}\right\rangle$ and the conditional expectation of some uncertain future profit over all load patterns in $C_{s}$. Linearization and subtraction of a term independent of $\boldsymbol{p}_{s}$ allows us to approximate the expected future profit by $L_{s}\left\langle\mathbf{1}_{d_{s}}, \boldsymbol{p}_{s}\right\rangle$, where $L_{s}$ represents the Lagrange multiplier associated with the energy balance equation of stage $s$ in problem (11). In economic terms, $L_{s}$ is the shadow price of energy. As it is part of the solution of (11), the $\mathcal{H}_{s}$-measurable random variable $L_{s}$ is a priori unknown. Let us now assume that period $s$ is much shorter than $\max \left\{\alpha_{1}, \alpha_{2}\right\}^{-1}$ for all $s \in \mathcal{S}^{+}$. This assumption is nonrestrictive for problems of practical relevance. Then, the deterministic vector

$\hat{\boldsymbol{F}}_{s}=E\left(\boldsymbol{F}_{s}\right)=\left(F\left(0, i_{s}\right), \ldots, F\left(0, i_{s+1}-1\right)\right)$,

characterizing the current forward curve for period $s$, is approximately parallel to the $\mathcal{H}_{s}$-measurable random vector $\boldsymbol{F}_{s}$ for any realization of the risk factors at the period start time. Concretely speaking, there is a $\mathcal{H}_{s^{-}}$ measurable normalization constant $M_{s}$ such that $\boldsymbol{F}_{s} \approx$ $M_{s} \hat{\boldsymbol{F}}_{s} \cdot{ }^{3}$ Assembling the above results, we see that a near-optimal strategy $\boldsymbol{p}_{s}$ for a certain scenario could principally be determined by solving the deterministic linear program

$\max _{\boldsymbol{p}_{s} \in C_{s}}\left\langle\hat{\boldsymbol{F}}_{s}-K_{s} \mathbf{1}_{d_{s}}, \boldsymbol{p}_{s}\right\rangle$

where $K_{s}$ is the realization of $\left(K-L_{s}\right) / M_{s}$ in that scenario. Although the random variable $\left(K-L_{s}\right) / M_{s}$ is completely unknown, the above argumentation shows that potentially optimal exercise profiles for period $s$ are generated by solving (13) for different values of $K_{s}$. Let us next introduce upper and lower bounds on the cur-

3 The components of the vectors $\hat{\boldsymbol{F}}_{s}$ and $\boldsymbol{F}_{s}$ are of the form $F(0, i)$ and $E\left(S(i) \mid \mathcal{H}_{s}\right)$, respectively, for $i_{s} \leq i<i_{s+1}$. Substituting $\boldsymbol{\xi}_{i}=H_{i, i_{s}} \boldsymbol{\xi}_{i_{s}}+\boldsymbol{\varepsilon}_{i, i_{s}}$ into (5) yields

$E\left(S(i) \mid \mathcal{H}_{s}\right)=F(0, i) \frac{\exp \left\langle\boldsymbol{e}_{1}, H_{i, i_{s}} \boldsymbol{\xi}_{i_{s}}\right\rangle}{E\left(\exp \left\langle\boldsymbol{e}_{1}, H_{i, i_{s}} \boldsymbol{\xi}_{i_{s}}\right\rangle\right)}$

where $\boldsymbol{e}_{1}=(1,0)$. By assumption, $\alpha_{1} d_{s} \ll 1$ and $\alpha_{2} d_{s} \ll 1$ for $d_{s}=i_{s+1}-i_{s}$. Using the results of Section 2, we thus find $H_{i, i_{s}}=I+O\left(\left(\alpha_{1}+\alpha_{2}\right) d_{s}\right)$, where $I$ stands for the two-dimensional identity matrix, and $O$ denotes the usual Landau symbol. Therefore, it is justified to approximate $H_{i, i_{s}}$ by $I$ in (12), which means that we may set $M_{s}=\exp \left(\xi_{1, i_{s}}\right) / E\left(\exp \left(\xi_{1, i_{s}}\right)\right)$. 
rent forward price curve for period $s$.

$K_{s}^{+}=\max \left\{F(0, i) \mid i=i_{s}, \ldots, i_{s+1}-1\right\}$

$K_{s}^{-}=\min \left\{F(0, i) \mid i=i_{s}, \ldots, i_{s+1}-1\right\}$

For any $K_{s} \leq K_{s}^{-}$the objective function coefficients in (13) are nonnegative, and the trivial 'maximum power strategy' achieves maximal profit. Conversely, for any $K_{s} \geq K_{s}^{+}$the objective function coefficients in (13) are nonpositive, and the trivial 'minimum power strategy' is optimal. Consequently, in order to generate interesting exercise profiles, it suffices to focus on those values of the control parameter $K_{s}$ which lie between $K_{s}^{-}$and $K_{s}^{+}$. In practice, one might wish to choose freely the number $n_{s} \geq 2$ of exercise profiles associated with period $s$. Subsequently, one should select a set of candidate parameters

$K_{s}^{-}=K_{s}^{1}<K_{s}^{2}<\cdots<K_{s}^{n_{s}}=K_{s}^{+}$,

and the exercise profile $\boldsymbol{p}_{s}^{n}$ can conveniently be taken to be some optimal solution of the linear program (13) for the parameter choice ${ }^{4} K_{s}=K_{s}^{n}, n=1, \ldots, n_{s}$. Without loss of generality, each exercise profile represents some vertex of $C_{s}$. Moreover, we may always postulate that the first and the last exercise profiles are given by the trivial minimum and maximum power profiles, respectively. These profiles prevent the pricing problem from becoming infeasible in the new formulation (11), i.e., the energy targets in problem (11) can always be met, provided the exact pricing problem (6) is feasible.

\subsection{Estimation of the Approximation Error}

In this section we will argue that our model facilitates the calculation of a tight lower bound on the option value. To this end, we will discuss the effects of the basic approximations that led from the exact pricing problem (6) to the numerically tractable stochastic program (11).

Problem (8) differs from the exact pricing problem (6) only in that the ramping constraints are disregarded at the period start times $i_{s}, s \in \mathcal{S}^{+}$, and the decisions are adapted to the reduced information process $\left\{\mathcal{H}_{s}\right\}_{s \in \mathcal{S}^{+}}$. Neglect of a small number of ramping constraints, on one hand, slightly increases the option value. This price appreciation can be estimated above by an error bound $\varepsilon>0$. For ease of exposition, assume that $\underline{p}_{i}=\underline{p}$ and $\bar{p}_{i}=\bar{p}$ are constant, while $\varrho_{i}=\varrho$ is a constant integer fraction of the power span $\bar{p}-p$ for all $i \in \mathcal{I}^{+}$. Then, a

4 If the forward curve exhibits prominent peaks, the choice of equidistant control parameters may lead to unsatisfactory results; especially for small values of $n_{s}$. In practice, one needs a suitable heuristics, which should depend both on the contractual specifications and the forward price data. valid error bound is given by

$\varepsilon=\frac{(\bar{p}-\underline{p})^{2}}{2 \varrho} \sum_{s \in \mathcal{S}^{+}} E\left(\left\|\boldsymbol{S}_{s}-K \mathbf{1}_{s}\right\|_{\infty}\right)$.

The first factor $(\bar{p}-p)^{2} / 2 \varrho$ estimates the maximum amount of energy which has to be bought or sold to match the profiles at the period start times. Moreover, $\left\|\boldsymbol{S}_{s}-K \mathbf{1}_{s}\right\|_{\infty}$ stands for the maximum net cost for buying or selling energy in period $s$. In a wellspecified model, where $(\bar{p}-\underline{p}) / \varrho$ is much smaller than $\min \left\{d_{s} \mid s \in \mathcal{S}^{+}\right\}$, the error bound $\varepsilon$ may be considered small. Note that (14) can be generalized to the case of time-dependent power limits and ratchets. The requirement that the decisions be adapted to the coarser information process $\left\{\mathcal{H}_{s}\right\}_{s \in \mathcal{S}^{+}}$, on the other hand, leads to a decrease of the option premium. This is a direct consequence of the fact that the measurability constraint in (8) is more restrictive than in (6). Thus, the optimal value of the exact pricing problem (6) is at least as large as the optimal value of (8) less $\varepsilon$. The outlined reduction of the information process is referred to as stage-aggregation in stochastic programming literature, cf. Birge and Louveaux (1997), Section 11.2. In a continuous time setting, it has been shown in Kuhn (2008) that the optimal value of the stage-aggregated problem converges to the optimal value of the original unaggregated problem if the stage durations become uniformly small. Due to extremely frequent exercise decisions, the original swing option pricing problem (6) behaves indeed like a continuous-time model. Therefore, the gap between the optimal values of problems (6) and (8) can be kept small by choosing suitably short stage durations.

Next, we discuss the discretization of the probability space. The objective of the stochastic program (8) is linear in the decision variables, and the coefficients are (at least) twice continuously differentiable in the risk factors. Following the recipe in Kuhn (2004), Section 5.1, one can therefore systematically construct a discrete probability measure $Q^{d}$ on $(\Omega, \mathcal{F})$ such that the optimal value of (8) is no smaller than the optimal value of the discretized problem (9) subtracted by some nonnegative constant $\varepsilon^{\prime}$, which depends on $Q^{d}$. This bound can be made arbitrarily tight. In fact, by successively refining the discrete measure $Q^{d}$, the optimum of (9) approaches the optimum of (8) while $\varepsilon^{\prime}$ converges to zero, cf. Kuhn (2004), Theorem 5.6. The final approximation step consists in a reparameterization of the decision space and a reduction of degrees of freedom. Thereby, the set of admissible exercise strategies is further restricted implying that the optimal value of (11) is no larger than the optimal value of $(9)$.

In summary, we conclude that the exact option premium is bounded below by the optimal value of the computationally tractable stochastic program (11) less the small constants $\varepsilon$ and $\varepsilon^{\prime}$. By the pertinent convergence results 
mentioned above, we expect the gap between the optimal values of problems (6) and (11) to be small if the number of stages as well as the number of discretization points and exercise profiles per stage are chosen adequately.

\section{Numerical Results}

Our numerical calculations are based on the Pilipovictype forward price model developed in Section 2. Given the relative immaturity of electricity markets, it is very difficult to obtain statistically significant estimates for the model parameters. In this paper, we completely sidestep the difficulties caused by parameter estimation. We merely remark that our swing option pricing model could be robustified against irreducible estimation errors, e.g. by evaluating the worst-case option premium over all volatility and mean-reversion parameters within a given confidence set. The development of a robust pricing problem is beyond the scope of this paper but constitutes a promising pathway for future research. Here, to obtain reasonable parameter values in a straightforward manner, we match the volatilities of the forward contracts to those observed on the EEX. Under the assumption that $\alpha_{2}=0$, this volatility matching is particularly simple. In fact, from (4) we see that $\sigma_{1}$ represents the volatility of the short-term forwards (with maturities $\ll \alpha_{1}^{-1}$ ), while $\sigma_{2}$ represents the volatility of the long-term forwards (with maturities $\gg \alpha_{1}^{-1}$ ). Note that the parameter $\alpha_{1}$ determines the boundary between short-term and long-term forwards. Based on these observations, we set $\alpha_{1}=6.849 \mathrm{E}-04 \mathrm{~h}^{-1}$, while the volatility coefficients are set to $\sigma_{1}=1.068 \mathrm{E}-02 \mathrm{~h}^{-1 / 2}$ and $\sigma_{2}=2.671 \mathrm{E}-03 \mathrm{~h}^{-1 / 2}$, respectively. The model must be initialized with the forward price curve observed at the swing option's valuation date. However, since only few futures contracts are traded in the market, the theoretical hourly forward price curve is observable only in a limited manner. To circumvent this problem we use a software package developed by Jens Güssow for constructing high-resolution forward price curves from available market data. This tool is based on a methodology due to Fleten and Flemming (2003): historical EEX spot price data is used to design a candidate term structure, which reflects the typical seasonal pattern. By applying a constrained least squares optimization scheme, the candidate curve is smoothed and adjusted to be consistent with currently observed EEX futures prices. Notice that the construction of the initial forward curve and the calibration of the stochastic forward price model are independent tasks. Electricity traders often have their own tools to construct forward price curves, integrating quantitative (measured data) and qualitative (expert opinion) information. Any such tool could be used to initialize our stochastic forward price model, which merely determines the random shifting and tilting of the initial curve over time.

Let us now turn to the valuation of some typical swing option contracts, all of which are derived from the base contract specified in Table 4. By convention, the valu-

Table 4

Parameters of the Base Contract

\begin{tabular}{rlrl}
\hline$\underline{t}=$ Aug. $12004,00: 00$ & $p_{\text {start }}$ & $=0 \mathrm{MW}$ \\
$\bar{t}$ & $=$ Oct. $312004,24: 00$ & $\underline{e}$ & $=0 \mathrm{MWh}$ \\
$\Delta$ & $=1 \mathrm{~h}$ & $\bar{e}$ & $=132 ' 480 \mathrm{MWh}$ \\
$K$ & $=20 € / \mathrm{MWh}$ & $\varrho_{i}$ & $=60 \mathrm{MW} \forall i \in \mathcal{I}$ \\
$\underline{p}_{i}$ & $=0 \mathrm{MW} \forall i \in \mathcal{I}$ & & $c^{+}=+\infty € / \mathrm{MWh}$ \\
$\bar{p}_{i}=60 \mathrm{MW} \forall i \in \mathcal{I}$ & $c^{-}$ & $=+\infty € / \mathrm{MWh}$ \\
\hline
\end{tabular}

ation date corresponds to the contract start date and may be set to $t=0$ after some suitable 'time translation'. Notice that the base contract's energy limits are redundant (as is the case in zone $C$ in Fig. 1). Thus, it reduces to a portfolio of cash, forwards, and European call options, and its value amounts to

$\sum_{i \in \mathcal{I}^{+}} \underline{p}_{i}(F(0, i)-K)+\left(\bar{p}_{i}-\underline{p}_{i}\right) C(0, i)$.

The current forward price curve $\{F(0, t)\}_{t>0}$ is known by assumption. Moreover, the time- 0 price $C(0, t)$ of a simple European call option with strike price $K$ and maturity $t$ can be calculated analytically. According to (5), the logarithm of the time- $t$ spot price is normally distributed under the risk-neutral measure $Q$, and its variance can be written as

$$
\begin{aligned}
\Sigma_{t}^{2}= & \frac{\sigma_{1}^{2}}{2 \alpha_{1}}\left(1-e^{-2 \alpha_{1} t}\right)+\frac{\alpha_{1}^{2}}{\left(\alpha_{1}-\alpha_{2}\right)^{2}}\left[\frac{\sigma_{2}^{2}}{2 \alpha_{2}}\left(1-e^{-2 \alpha_{2} t}\right)\right. \\
& \left.+\frac{\sigma_{2}^{2}}{2 \alpha_{1}}\left(1-e^{-2 \alpha_{1} t}\right)-\frac{2 \sigma_{2}^{2}}{\alpha_{1}+\alpha_{2}}\left(1-e^{-\left(\alpha_{1}+\alpha_{2}\right) t}\right)\right] .
\end{aligned}
$$

Thus, ordinary call options are priced via the BlackScholes-type formula

$$
\begin{aligned}
C(0, t)= & F(0, t) \Phi\left(\left[\ln \frac{F(0, t)}{K}+\frac{\Sigma_{t}^{2}}{2}\right] / \Sigma_{t}\right) \\
& -K \Phi\left(\left[\ln \frac{F(0, t)}{K}-\frac{\Sigma_{t}^{2}}{2}\right] / \Sigma_{t}\right),
\end{aligned}
$$

where $\Phi$ stands for the standard normal distribution function. Substituting the parameter values of Table 4 into (15) and (16), we can analytically evaluate the fair price of the base contract, which amounts to $1.426 \mathrm{E}+06 €$.

If the base contract is assigned restrictive energy limits or nontrivial ratchets, analytical tractability is lost, and a numerical valuation scheme is needed. Numerical calculations are based on the approximations described in Section 5. First, the hourly rebalancing intervals within the 3 -month contract period are aggregated to a small 

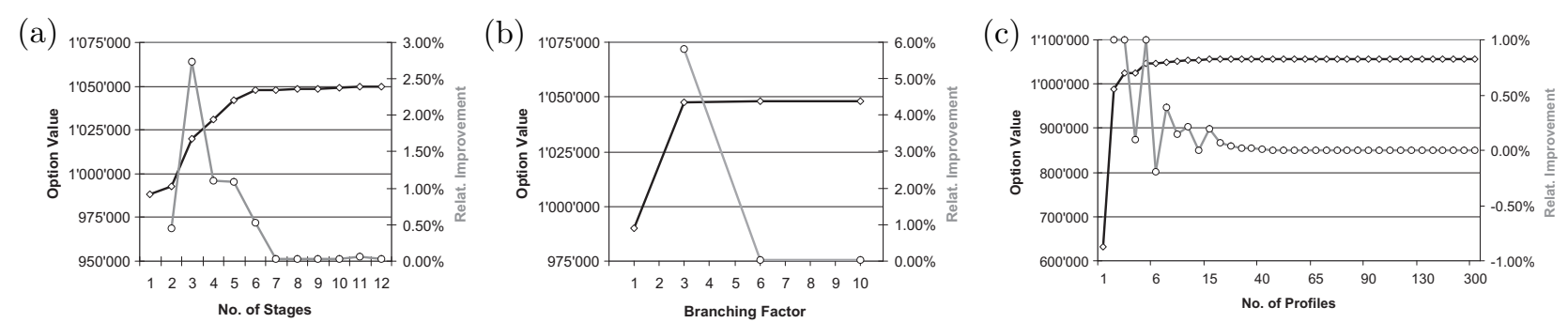

Fig. 2. Convergence of the three approximations number of stages. The first stage, which will provide deterministic exercise decisions, encompasses one week, whereas the remaining stages are of equal length. Requiring the first stage to comprise at least one week is reasonable since (deterministic) exercise schedules must be announced several days ahead in practice. On the other hand, the first stage should not exceed one week since a fine temporal granularity at the beginning of the planning horizon typically improves the quality of the first stage decisions. Next, the probability space is discretized by means of the moment matching method proposed in Siede (2000), Section 7.3; see Section 5.2 for more details. The corresponding scenario tree has a constant branching factor (number of branches per node). Finally, the decision space is reparameterized by concentrating on convex combinations of a small number of exercise profiles within each decision stage. We solved problem instances with up to 12 effective decision stages, up to 300 exercise profiles, and with branching factors equal to $1,3,6$, or 10 . Our numerical experiments indicate that approximate stochastic programs with 6 effective stages, 3 branches per node, and 6 exercise profiles achieve reasonable accuracy. Unless otherwise noted, all computational result reported below are based on this parameter choice. In this case, we observe solution times of less than 4 seconds, ${ }^{5}$ which ensures that our tool can be used by energy traders for real-time price negotiations over the telephone. The numerical value of the base contract amounts to $1.420 \mathrm{E}+06 €$, thus underestimating the analytical value by $0.4 \%$. This underestimation is consistent with the finding that our algorithm provides a lower bound on the option premium. Moreover, the high level of precision encountered in this special case suggests that our valuation scheme generally performs well. Note that we could still improve precision at the cost of additional runtime. If nontrivial energy limits or ramping constraints are introduced, the contract becomes path-dependent and analytically intractable. In order to estimate the accuracy of our valuation scheme for general path-dependent contracts, we perform a numerical convergence analysis based on a modified base contract with $\bar{e}=52^{\prime} 992 \mathrm{MWh}$. Note that this contract

\footnotetext{
5 All calculations were carried out on a $1 \mathrm{GHz}$ Pentium III PC with $512 \mathrm{MB}$ storage, and the instances of problem (11) were solved with CPLEX 9.1.
}

may be exercised at maximum power only during $40 \%$ of the planning horizon since $\bar{e}=\bar{p}(\bar{t}-\underline{t}) \times 40 \%$. Figure 2 shows convergence of the swing option value as (a) the number of effective decision stages, (b) the branching factor, and (c) the number of exercise profiles are increased. The number of stages is fixed to 6 in (b) and (c), the branching factor is fixed to 3 in (a) and (c), and the number of exercise profiles is fixed to 6 in (a) and (b). We observe monotonic convergence from below in all three cases. The saturation of the option value supports our hypothesis that the used approximations are tight. Moreover, the observed monotonicity reflects our intuition that the approximate pricing problem (11) provides an increasingly accurate lower bound on the optimal value of (6) when any of the underlying approximations is improved, see Section 5.

Figure 2 supports our assertion that approximations based on 6 effective stages, 3 branches per node, and 6 exercise profiles are reasonably accurate. Problem size grows exponentially with the number of stages and polynomially with the number of branches per node. Thus, any increase of the number of stages or the branching factor is computationally expensive. However, problem size grows only linearly with the number of decision variables per node, and introducing additional exercise profiles is therefore relatively cheap in terms of computation time. For illustrative purposes, we consider several interesting variants of the base contract. On one hand, we reduce the upper energy limit in steps of $10 \%$. On the other hand, we introduce uniform ratchets, which are integer fractions of the power span $\bar{p}_{i}-\underline{p}_{i}$. Both modifications entail a successive decrease of the option value, see Fig. 3. If the ratchets tend to zero, the exercise strategy $\left\{p_{i}\right\}_{i \in \mathcal{I}^{+}}$ becomes constant, and since start power is set to $0 \mathrm{MW}$, the swing option becomes worthless. However, for very small ratchets our numerical valuation scheme becomes obsolete as the ramping constraints are disregarded at the period start times. The forward price curve used in our calculations is visualized in the top diagram of Fig. 4. Having solved the approximate pricing problem (11), an efficient exercise strategy for the first contract week is 


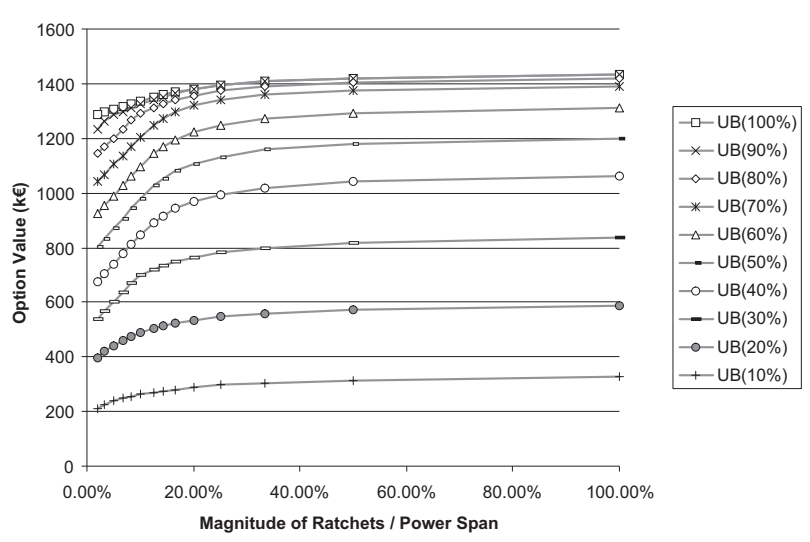

Fig. 3. Option value in dependence of the magnitude of the ratchets and for different values of the upper energy limit
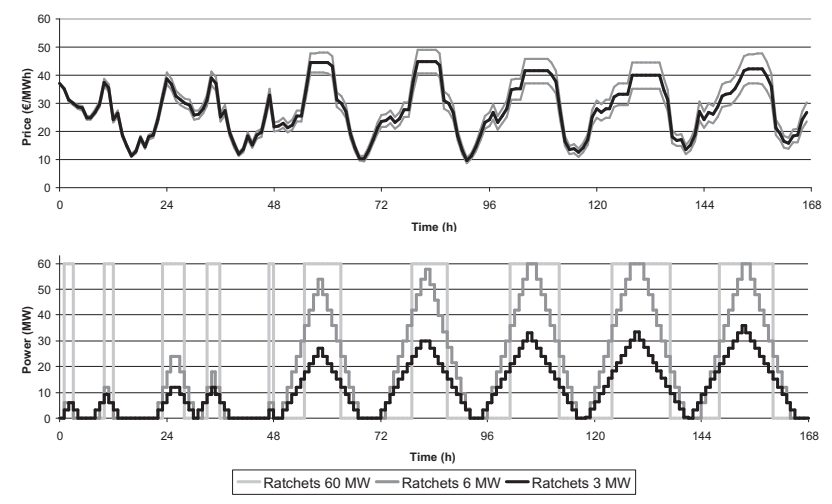

Fig. 4. Top: expected value and $67 \%$ confidence interval of the risk-neutral spot price distribution in the first contract week; bottom: exercise strategies for three contracts with increasingly restrictive ramping constraints

found by solving the deterministic problem

$$
\left.\begin{array}{ll}
\max & \sum_{i=0}^{d_{1}-1}(F(0, i)-K) p_{i} \\
\text { s.t. } & \sum_{i=0}^{d_{1}-1} p_{i}=e_{0}^{*} \\
& \underline{p}_{i} \leq p_{i} \leq \bar{p}_{i} \\
& \left|p_{i}-p_{i-1}\right| \leq \varrho_{i}
\end{array}\right\} \forall i=0, \ldots, d_{1}-1 .
$$

Here, the optimal first-stage decision $e_{0}^{*}$ of the stochastic pricing problem (11) enters the constraints of (17) as a parameter and specifies the energy target for the first contract week. Moreover, the dummy variable $p_{-1}$ is set to the start power $p_{\text {start }}$. Notice that the short term optimization (17) ensures consistency with the contractual provisions and the energy target provided by (11). Furthermore, it guarantees optimality with respect to the current forward price curve. In practice it is indeed desirable to evaluate a deterministic short term strategy since exercise schedules must usually be submitted (and thus known) several days ahead.

The bottom diagram in Fig. 4 shows the short term exercise strategies for three modified base contracts with different ratchets and a 50\% upper energy limit.

\section{Conclusions}

In this article we study a rich family of electricity swing options. We formulate an exact pricing scheme in a Pilipovic-type model economy driven by two exogenous risk factors. Notice, however, that our pricing scheme is flexible enough to cope easily with a larger number of risk factors. In order to convert the exact pricing model to a computationally tractable stochastic program, we apply three fundamental approximations: aggregation of decision stages, discretization of the probability space, and reparameterization of the decision space. The suggested approach facilitates calculation of a tight lower bound on the option premium. Investigation of analytically tractable limiting cases indicates that our methodology achieves a high degree of precision. As opposed to classical dynamic programming or Least Squares Monte Carlo techniques, our stochastic programming approach performs well in the presence of various risk factors and state variables. It applies even to contracts with a high number of exercise times (such as hourly exercisable swing options with a delivery period of one year). Our model easily accommodates new contractual provisions and additional constraints. Future research will be concerned with the determination of a tight upper bound on the option premium and generalization of the presented pricing scheme to real pumped hydropower plants.

\section{Acknowledgements}

We thank Jens Güssow for the permission to use his tool for constructing forward price curves. Moreover, we thank Patrick Wirth for stimulating discussions.

\section{References}

Ash, R. (1972). Real Analysis and Probability. Probability and Mathematical Statistics. Academic Press, Berlin.

Barbieri, A., \& Garman, M. (1996). Understanding the valuation of swing contracts. In Energy and Power Risk Management.

Birge, J., \& Louveaux, F. (1997). Introduction to Stochastic Programming. Springer-Verlag, New York.

Carmona, R., Dayanik, S. (2008). Optimal multiplestopping of linear diffusions and swing options. Mathematics of Operations Research, 33(2), 446-460.

Carmona, R., \& Ludkovski, M. (2005). Gas storage and supply guarantees: an optimal switching approach. Working Paper, Princeton University. 
Carmona, R., \& Touzi, N. (2008). Optimal multiple stopping and valuation of swing options. Mathematical Finance, 18(2), 239-268.

Davison, M., \& Anderson, L. (2003). Approximate recursive valuation of electricity swing options. Technical Report, The University of Western Ontario.

Deng, S., Johnson, B., \& Sogomonian, A. (1998). Exotic electricity options and the valuation of electricity generation and transmission assets. Decision Support Systems 30, 383-392.

Doege, J., Schiltknecht, P., \& Lüthi, H.-J. (2006). Risk management of power portfolios and valuation of flexibility. OR Spectrum 28(2), 267-287.

Fleten, S.-E., \& Lemming, J. (2003). Constructing forward price curves in electricity markets. Energy Economics 25(5), 409-424.

Ibáñez, A. (2004). Valuation by simulation of contingent claims with multiple exercise opportunities. Mathematical Finance 14(2), 223-248.

Jaillet, P., Ronn, E., \& Tompaidis, S. (2004). Valuation of commodity based swing options. Management Science 50(7), 909-921.

Kaminski, V., \& Gibner, S. (1995). Exotic options. In Managing Energy Price Risk, Kaminski, V. (Ed.). Risk Publications, London (pp. 117-148).

Kaut, M., \& Wallace, S. W. (2007). Evaluation of scenario generation methods for stochastic programming. Pacific Journal of Optimization 3(2), 257-271.

Keppo, J. (2004). Pricing of electricity swing options. The Journal of Derivatives 2(11), 26-43.

Kuhn, D. (2004). Generalized Bounds for Convex Multistage Stochastic Programs, vol. 548 of Lecture Notes in Economics and Mathematical Systems. SpringerVerlag, Berlin.

Kuhn, D. (2008). An information-based approximation scheme for stochastic optimization problems in continuous time. To appear in Mathematics of Operations Research.

Lari-Lavasanni, A., Simchi, M., \& Ware, A. (2001). A discrete valuation of swing options. Canadian Applied Mathematics Quarterly 9(1), 35-74.

Longstaff, F., \& Schwartz, E. (2001). Valuing american options by simulation: A simple least squares approach. The Review of Financial Studies 14(1), 113147.

Meinshausen, N., \& Hambly, B. (2004). Monte Carlo methods for the valuation of multiple exercise options. Mathematical Finance 14(4), 557-583.

Pflug, G. (1996). Optimization of Stochastic Models. Kluwer Academic Publishers, Hingham, MA.

Pilipovic, D. (1998). Energy Risk: Valuing and Managing Energy Derivatives. McGraw-Hill, New York.

Pilipovic, D., \& Wengler, J. (1998). Getting into the swing. Energy and Power Risk Management 2.

Protter, P. (1990). Stochastic Integration and Differential Equations: a New Approach. Springer-Verlag, New York.

Rockafellar, R., \& Wets, R.-B. (1976). Stochastic convex programming: Relatively complete recourse and induced feasibility. SIAM Journal on Control on Optimization 14, 574-589.

Siede, H. (2000). Multi-Period Portfolio Optimization. $\mathrm{PhD}$ thesis, University of St. Gallen.

Thompson, A. (1995). Valuation of path-dependent contingent claims with multiple exercise decisions over time: The case of take-or-pay. Journal of Financial and Quantitative Analysis 30(2), 271-293.

Thompson, M., Davison, M., \& Rasmussen, H. (2004). Valuation and optimal operation of electric power plants in competitive markets. Operations Research 52(4), 546-562. 